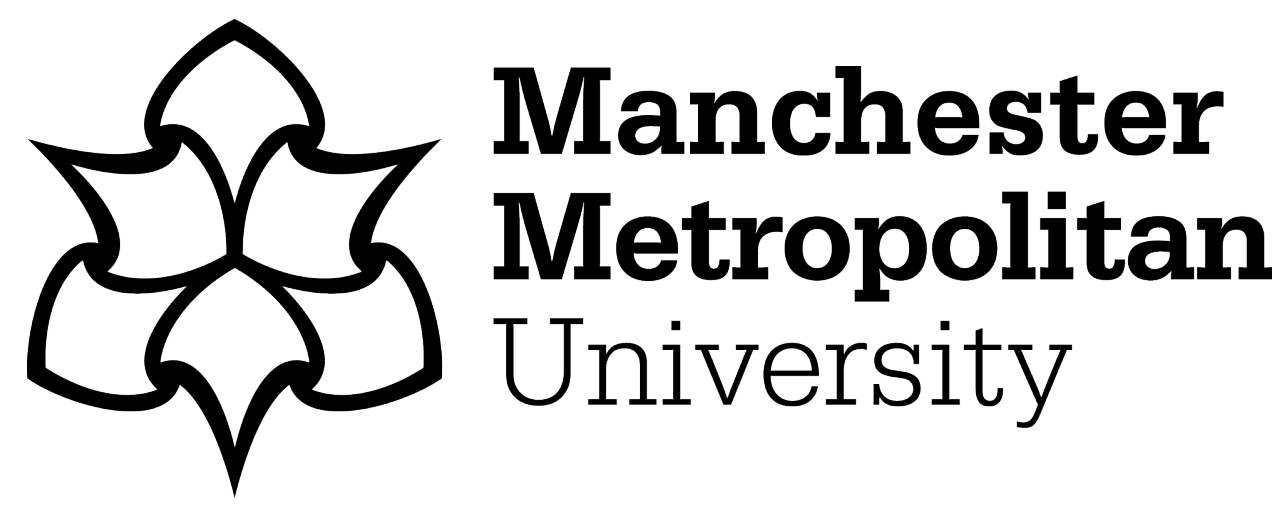

Tournier, I ORCID logoORCID: https://orcid.org/0000-0003-0403-4876, Marie-Frédérique Vives and Postal, V (2017) Animal-Assisted intervention in dementia: Effects on neuropsychiatric symptoms and on caregivers' distress perceptions. Swiss Journal of Psychology, 76 (2). pp. 51-58. ISSN 1421-0185

Downloaded from: https://e-space.mmu.ac.uk/627747/

Version: Accepted Version

Publisher: Hogrefe

DOI: https://doi.org/10.1024/1421-0185/a000191

Please cite the published version 


\title{
Animal-Assisted Intervention in Dementia
}

\section{Effects on Neuropsychiatric Symptoms and on Caregivers' Distress Perceptions}

\author{
Isabelle Tournier ${ }^{1}$, Marie-Frédérique Vives ${ }^{2}$, and Virginie Postal ${ }^{3}$ \\ ${ }^{1}$ Integrative Research Unit on Social and Individual Development, University of Luxembourg, Esch-sur-Alzette, Luxembourg \\ ${ }^{2}$ EHPAD les Jardins de Jovinius, Jonzac, France \\ ${ }^{3}$ Laboratoire de Psychologie Santé et Qualité de Vie, University of Bordeaux, France
}

\begin{abstract}
The present work assesses the efficacy of an animal-assisted therapy (AAT) program in the reduction of neuropsychiatric symptoms in older adults with medium to severe dementia. Performed in an Alzheimer's disease/dementia care unit, the intervention included 11 elderly residents aged 71 to 93 years (mean age $=82.91$ years; mean Mini-Mental State Examination score $=7.8 / 30$ ). Behaviors during the AAT sessions as well as pre/post intervention neuropsychiatric symptoms were examined during this 5-month weekly intervention conducted by an AATcertified psychologist along with her dog. AAT had a positive effect on total score and caregiver distress score for several neuropsychiatric symptoms (i.e., delusion, depression, disinhibition, euphoria, and aberrant motor activity). Moreover, the ratings of the various behaviors during each session suggest that the beneficial effects of AAT appear during the first few sessions. These results support the notion that regular and long-term AAT sessions are an effective alternative to pharmacological interventions for the reduction of neuropsychiatric symptoms.
\end{abstract}

Keywords: animal-assisted therapy, severe dementia, behavioral and psychological symptoms

Animal-assisted therapy (AAT) aims to improve or maintain a patient's physical, psychological, and/or sociological functioning (Kruger \& Serpell, 2006) by influencing the interaction between the therapist, an animal (usually a dog, but also horses, birds, cats, etc.), and one or more patients. Research on the effectiveness and best practices of AAT is on the rise (Fine, 2010), and it has been suggested that pets are an effective object of attachment for many humans (Levinson, 1969; Zilcha-Mano, Mikulincer, \& Shaver, 2011) as well as a beneficial source of sensory and physical stimulations, reminiscences, and social interaction (Filan \& Llewellen-Jones, 2006). Further advantages of using pets in therapy are that they are nonjudgmental, and that they rely on body language more than humans do, which is especially valuable when working with patients with impaired language skills (Filan \& Llewellen-Jones, 2006; Tribet, Boucharlat, \& Myslinski, 2008).

Thus, AAT is particularly suited for older adults with senile dementia. Alzheimer's disease (AD) and senile dementia in general are characterized by physical and cognitive decline as well as psychological and relational problems (Epple, 2002). The prevalence of neuropsychiatric symptoms such as apathy, depression, agitation, and anxiety is high among people with $\mathrm{AD}$ and related dementias. Some $75 \%$ to $90 \%$ of nursing-home patients with dementia show at least one behavioral alteration, and this prevalence increases with growing severity of the dementia (Selbaek, Engedal, \& Bergh, 2013). This very high prevalence is worrying given that these neuropsychiatric symptoms contribute to patient and caregiver distress (Cummings \& McPherson, 2001; Wood et al., 1999). At present, behavioral alterations are usually treated by psychotropic medications, which have an unclear efficacy and frequent adverse effects (Ballard \& Corbett, 2010), with sudden death as a critical issue (Pollock \& Mulsant, 2011). Consequently, the nonpharmacological management of neuropsychiatric symptoms is a high priority for practitioners as well as patients and their families. AAT has been found to have a positive impact on treating depression, agitation, and aggressiveness in older adults with dementia (for meta-analyses, see Bernabei et al., 2013; Virués-Ortega, Pastor-Barriuso, Castellote, Población, \& de Pedro-Cuesta, 2012). However, the positive effects observed are not systematic (Motomura, Yagi, \& Ohyama, 2004) or may last for only a short period of time (Richeson, 2003). In addition, the interventions studied are often presented in a time-intensive format (i.e., several times a week; see Bernabei et al., 2013), which does not necessarily reflect the format of nonpharmacological interventions in nursing homes due to staff, time, and resource constraints (e.g., van der Ploeg, Mbakile, Genovesi, \& O’Connor, 2012).

Currently, AAT constitutes an interesting and promising nonpharmacological alternative, especially given that activities suitable for older adults with dementia are scarce because of their illness-related impairments. Comparing general behavior ratings before and after AAT sessions is the most frequently used method to examine the benefits of AAT in older adults 
with dementia. A few studies observed these patients' behaviors during AAT sessions and reported a substantial drop in noise levels in the ward (Walsh, Mertin, Verlander, \& Pollard, 1995) as well because ore alert and responsive behavior during the sessions (Richeson, 2003). However, to our knowledge, none of the previous studies examining the effectiveness of AAT measured changes during the AAT sessions as well as compared behavior before and after the sessions. Moreover, it remains an open question whether behavior continues to improve with an increasing number of AAT sessions. Specifically, do the effects of AAT increase with increasing number of AAT sessions or do the effects remain stable after a certain number of sessions? Regarding mood, in a sample of institutionalized older adults, Crowley-Robinson, Fenwick, and Blackshaw (1996) found that AAT had a positive effect over a 15-month intervention period, with mood gradually improving with increasing number of sessions.

The present pilot study assessed the efficacy of an AAT program implemented in a nursing home to reduce neuropsychiatric symptoms in older adults with medium to severe dementia and to study the progression of changes during the course of AAT. To this end, we investigated behaviors during the AAT sessions and neuropsychiatric symptoms both prior to and after 5 months of a weekly AAT intervention. We hypothesized that, with increasing number of sessions, the frequency of positive behaviors (e.g., smiling, relaxed), interaction with the dog (e.g., watching the dog, petting the dog), and participation (e.g., evoking memories, physical involvement) would increase, while the frequency of negative behaviors (e.g., anxiety, sadness) would decrease. Moreover, we hypothesized that the intensity and severity of reported neuropsychiatric symptoms as well as their impact on caregiver distress would be lower after the AAT intervention.

\section{Method}

\section{Participants and Intervention}

The AAT intervention was implemented in the care unit of a French nursing home dedicated to residents with severe Alzheimer's disease or other types of senile dementia. This intervention resulted from the nursing home's desire to offer a nonpharmacological intervention aimed at reducing the frequency of behavioral disturbances in their patients with Alzheimer's disease. AAT intervention was suggested as a viable nonpharmacological intervention by the staff psychologist specialized and trained in AAT. This intervention followed the ethical guidelines outlined in the Declaration of Helsinki and was approved by the Ethics Review Board of the University of Bordeaux and the nursing home directory staff. Informed consent was obtained from all participants and their legal guardians.
Before each session, participants were reminded that they could withdraw their consent or discontinue participating in the sessions temporarily or permanently at any time and for any reason. In addition, during the sessions with the therapy animal (i.e., a dog) the psychologist continuously assessed whether participants presented any signs of fear or rejection. Thus, the present pilot study reports the empirical evaluation of this AAT intervention in a sample of institutionalized older adults with dementia.

All 15 residents of the unit agreed to participate in the AAT intervention, the majority of whom reported having had animals (e.g., pets, farm animals) in the distant or more recent past. Of the 15 participants, one died and one left the unit during the study period, and the data of two others could not be included in the final analysis because of their infrequent participation (less than $60 \%$ of the 19 sessions), which they stated resulted from family visits or tiredness. Consequently, the final sample included 11 residents (10 females, 1 male) aged 71 to 93 years (mean age $=82.91$ years). The participants' mean Mini-Mental State Examination score (MMSE Greco version; Kalafat, Hugonot-Diener, \& Poitrenaud, 2003) was 7.8 (of 30 possible points), indicating severe dementia. On the basis of medical, neurological, and psychological examinations, $73 \%$ of the participants were diagnosed by the geriatric physician and the unit psychologist with severe dementia, whereas the others were diagnosed with moderately severe dementia (see participants' characteristics in Table 1).

Table 1. Participants' characteristics

\begin{tabular}{lllll}
\hline Participant & Sex & Age & MMSE (/30) & $\begin{array}{l}\text { Estimated severity } \\
\text { of dementia }\end{array}$ \\
\hline 1 & Female & 71 & 15 & Moderately severe \\
2 & Female & 82 & 13 & Moderately severe \\
3 & Female & 93 & 13 & Moderately severe \\
4 & Female & 85 & 12 & Moderately severe \\
5 & Female & 90 & 7 & Severe \\
6 & Male & 73 & 4 & Severe \\
7 & Female & 80 & 3 & Severe \\
8 & Female & 80 & 3 & Severe \\
9 & Female & 83 & 0 & Severe \\
10 & Female & 84 & Refusal & Severe \\
11 & Female & 91 & Refusal & Severe \\
\hline
\end{tabular}

AAT sessions took place once a week (on Tuesdays) at the same time for a duration of approximately 1 hour. This format appears typical of the regular interventions implemented in nursing homes and affords a high level of ecological validity to the results. Based on the literature on AAT in patients with Alzheimer's disease, we determined that 5 months was the appropriate length of time to study possible therapy-related changes. The AAT team was composed of the psychologist, her own dog, and at least one member of the nursing staff. The psychologist was certified in AAT and had completed several training pro- 
grams with her dog. Her dog was a 7-year-old Labrador retriever that had satisfied several canine behavior requirements (e.g., was sociable, nonaggressive, calm) and was a certified therapy dog. This trainer-dog pair had had several previous AAT experiences in nursing homes and units for disabled people. Nursing staff assistants included one nurse and two nurse's aides who were highly familiar with the handling of animals and who had expressed interest in assisting with the intervention. Their participation varied according to their work schedules. During the weekly sessions, participants were encouraged to interact with and take care of the dog (e.g., to pet, brush, feed, talk to her, etc.) as well as to recall prior memories that included animals.

\section{Measurement}

The measures included a systematic assessment of behaviors during the first 19 AAT sessions ${ }^{1}$ (November 2013 to March 2014). In addition, a baseline and immediate follow-up evaluation of neuropsychiatric symptoms was carried out.

\section{Behavior During AAT Sessions}

At the end of each session, using a list of 21 behaviors, the psychologist-staff member team rated the behavior displayed by the participants during the AAT sessions. Their observations were assigned to one of four categories: negative affect (comprising anxious, sad, aggressive, and apathetic behavior), positive affect (comprising focused, smiling, relaxed, and pleasure behavior), interaction with the dog (comprising watching, touching, holding, petting, brushing, playing with, walking, and feeding the dog), and participation during the session (comprising spontaneous participation, evoked memories, physical involvement, verbal communication, and showing interest in the other participants). Thus, the maximum scores attainable for each participant at each session were four for negative affect, four for positive affect, eight for interaction with the dog, and five for participation during the session. We calculated average behavior scores for each category in each of four time periods: Period 1 (Sessions 1 to 5), Period 2 (Sessions 6 to 10), Period 3 (Sessions 11 to 15 ), and Period 4 (Sessions 16 to 19). We grouped the sessions to provide a general overview of temporal changes, but reduce the influence of variability on the general pattern of change.

\section{Neuropsychiatric Symptoms}

The severity of neuropsychiatric symptoms, measured with the Neuropsychiatric Inventory (NPI; Cummings et al., 1994), was assessed by the head nurse of the unit (after consultation with the nurse's aides) on two occasions: immediately before the 19 AAT sessions began and after they ended. This widely used global assessment scale originally consisted of 10 items evalu- ating delusions, hallucinations, depression/dysphoria, anxiety, agitation/aggression, euphoria, disinhibition, irritability/lability, apathy, and aberrant motor activity. Later, two supplementary items on sleep/nighttime behavior and appetite/eating disorders were added. The caregiver-respondents were to select behaviors that had been present during the previous four weeks, but had not been characteristic of the patient before the onset of dementia. The respondents also rated the frequency of the selected behaviors on a scale from 1 (less than once a week) to 4 (once or more per day or continuously) and their severity on a scale from 1 (mild) to 3 (severe). A composite score (total score) was calculated for each of the 12 items by multiplying frequency (maximum score $=4$ ) by severity (maximum score $=3$ ), which resulted in a maximum score of 12 . Moreover, the caregivers rated their emotional and/or psychological distress linked to neuropsychiatric symptoms on a scale from 0 (not at all) to 5 (very severe/extreme). The addition of this composite score for the 12 assessed behaviors constitutes the total score of the NPI, and the validity and reliability of the scale are satisfactory (Cummings \& McPherson, 2001). In the present study, we used the French translation (Sisco et al., 2000) of the nursing home version of the NPI (NPI-NH; Wood et al., 2000).

\section{Statistical Analysis}

The normality and sphericity of the data were checked, and square root transformations were applied only to negative affect scores because the normality of the data needed improvement. Regarding observed behaviors during AAT sessions, repeated measures ANOVAs (Period 1, Period 2, Period 3, and Period 4) were performed for each of the four behavioral categories (negative affect, positive affect, interaction with the dog, and participation). First, the total NPI scores (total score and distress score) before and after the intervention were compared with paired samples $t$-tests for the total score in a first time. Cohen's $d$ were performed to estimate effect size. Second, the same NPI scores were compared separately for each of the 12 items.

\section{Results}

\section{Observed Behaviors During AAT Sessions}

In Figure 1, the means of the behaviors observed during AAT sessions are presented as a function of period $(1,2,3$, and 4) and behavioral category (negative affect, positive affect, interaction with the dog, and participation).

1 Initially, 20 sessions were planned but one had to be deleted due to a 1-week sick leave of the psychologist. 


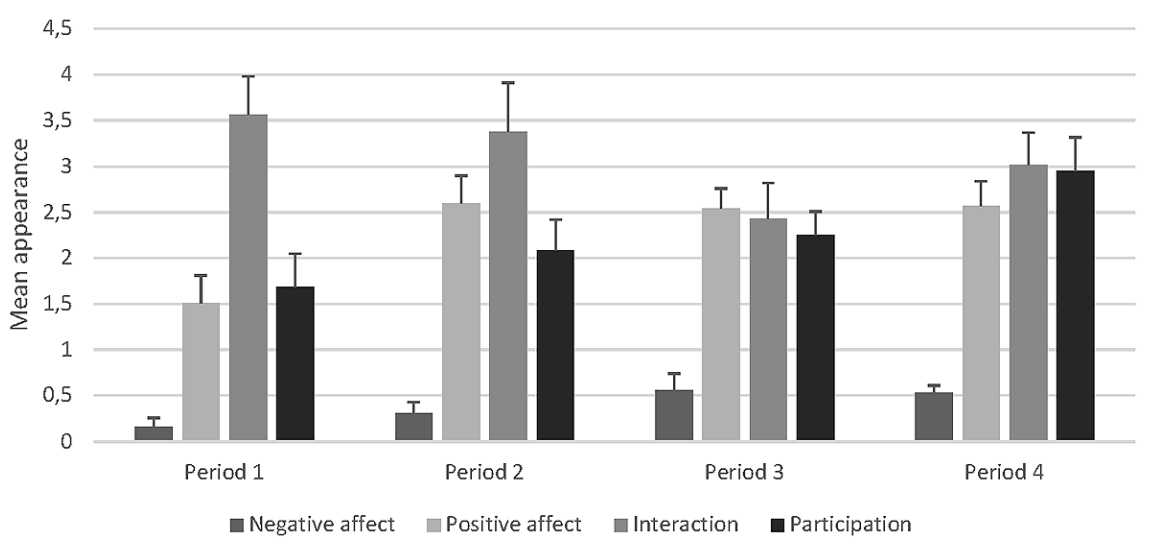

Figure 1. Mean appearance and standard errors of observed behavior during AAT sessions as a function of period (1, 2, 3, and 4) and behavior category (negative affect, positive affect, interaction with the dog, and participation).

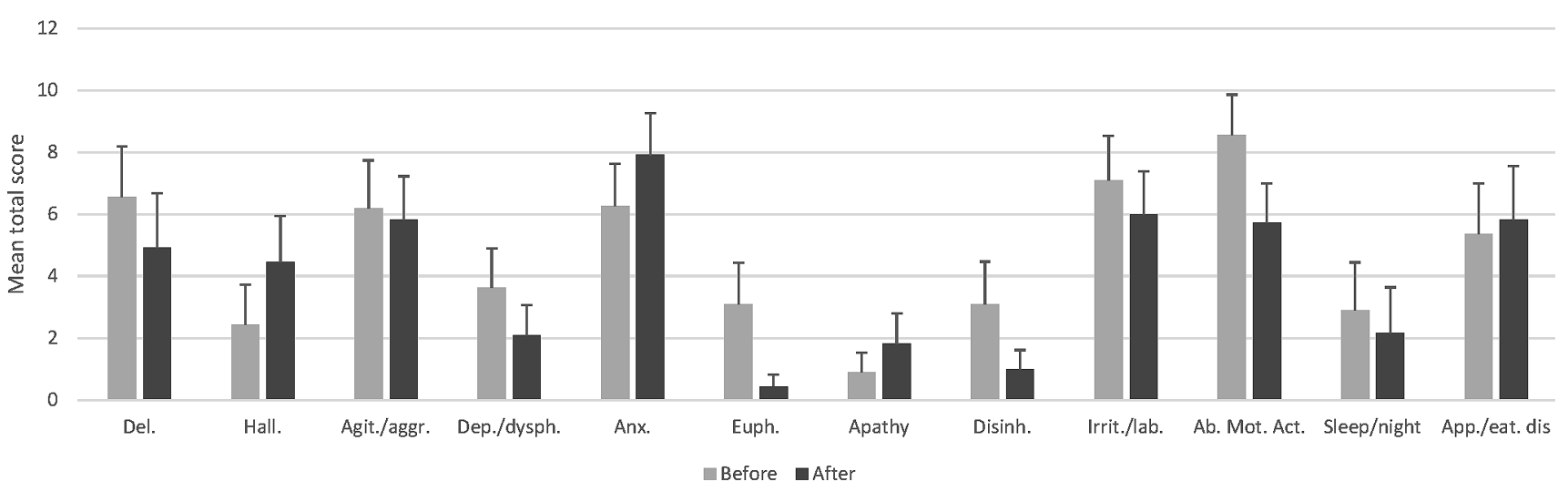

Figure 2. Mean total scores and standard errors before and after AAT intervention for each Neuropsychiatric Inventory item. Del. = delusion; Hall. = hallucination; Agit./aggr. = agitation/aggressivity; Dep./dysph. = depression/dysphoria; Anx. = anxiety; Euph. = euphoria; Disinh. = disinhibition; Irrit./lab. = irritability/lability; Ab. mot. act. = aberrant motor activity; Sleep/night. = sleep/nighttime disorder; App./eat. dis. = appetite/eating disorder.

Repeated ANOVAs for each period were performed separately for each behavioral category. The effect of AAT session period was significant for all categories investigated and is described below. Regarding negative affect, a global increase was observed, $F(3,30)=2.91, p=.05, \eta_{p}^{2}=0.22$. Tests of contrast revealed a significant increase in negative affect between Periods 1 and $3(p=.001)$ and Periods 1 and $4(p=.001)$. The difference between Periods 1 and 2 and between Periods 2 and 3 was nonsignificant, but that between Periods 2 and 4 was significant $(p=.04)$. As for positive affect, there was a significant effect of period, $F(3,30)=5.50, p=.004, \eta^{2}{ }_{p}=0.35$, with a significant increase between Period 1 and all others periods (Period 2: $p=.006$, Period 3: $p=.001$, Period 4: $p=.03$ ), but there was no significant difference between Periods 2, 3, and 4. Regarding interactions, the significant effect revealed a decrease, $F(3,30)=3.66, p=.02, \eta^{2}{ }_{p}=0.27$, that was only significant between Periods 1 and $3(p=.001)$; the other differences between periods were nonsignificant. Finally, participation increased, $F(3,30)=5.45, p=.004, \eta^{2}{ }_{p}=0.35$, with significant increases between Periods 1 and $3(p=.03)$, Periods 2 and $4(p=.03)$, and Periods 1 and $4(p=.03)$, but a nonsignificant difference between Periods 3 and 4 .

\section{Pre/Post Intervention Comparisons of the NPI Scores}

Compared to the baseline measure, the NPI total score after the intervention showed a decrease, though it was not statistically significant $(56.09 \text { vs. } 48.18, t(10)=1.18, p=.27)^{2}$. The same pattern was observed for the caregiver distress score $(23.18$ vs. $17.82, t(10)=1.79, p=.10)$.

The means of the pre/post intervention NPI total score comparisons for each of the 12 behaviors are presented in Figure 2. Changes were found in several score comparisons after the AAT intervention. Specifically, we found an increase in hallucination (2.45 vs. 4.45), $t(10)=-1.91, p=.08, d=-.38$, a decrease in depression (3.64 vs. 2.09), $t(10)=1.87, p=.09$, and $d=.41$, a decrease in euphoria (3.09 vs. 0.45$), t(10)=1.87$, $p=.09$, and $d=.81$, and a decrease in aberrant motor activity, (8.55 vs. 5.73 ), $t(10)=2.10, p=.06$, and $d=.65$. All other $p$ values were greater than .10 .

The means of the pre/post intervention NPI distress scores for each of the 12 behaviors are presented in Figure 3. Three comparisons revealed a significant decrease in distress: depres-

\footnotetext{
2 In this composite score, the decrease was larger for severity $(16.45$ vs. 13.36$), t(10)=1.69, p=.12$, than frequency $(22.82$ vs. 21.09$)$, $t(10)=0.74$, $p=.47$.
} 


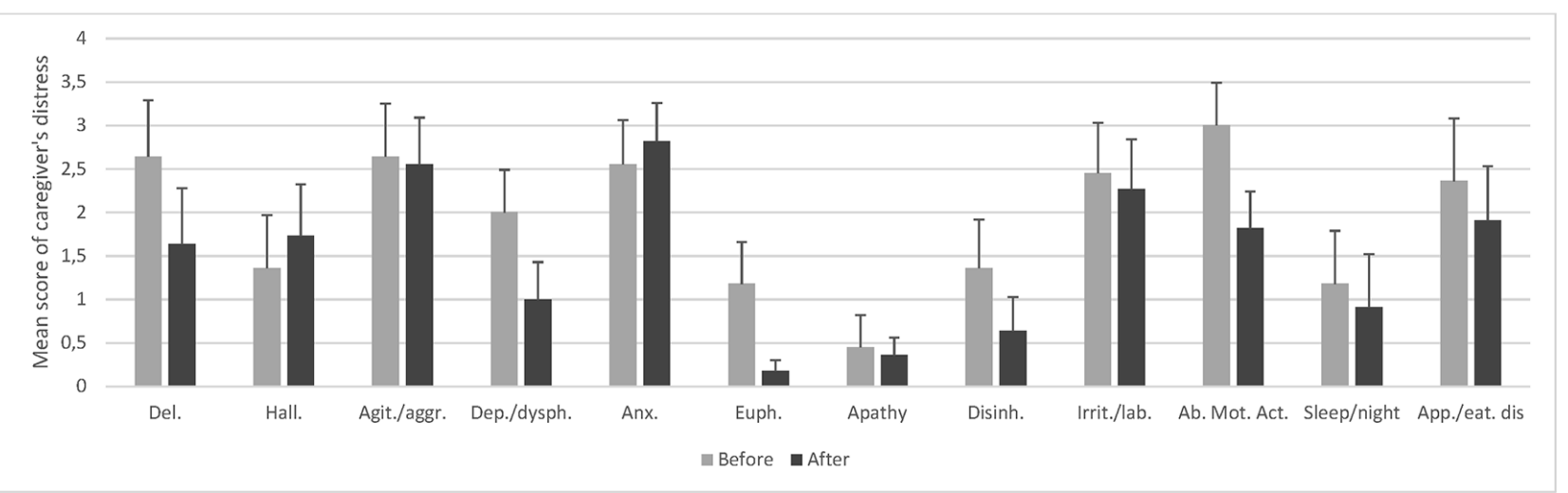

Note. Del. = delusion, Hall. = hallucination, Agit./aggr. = agitation/aggressivity, Dep./dysph. = depression/dysphory, Anx. = anxiety, Euph. $=$ euphoria, Disinh. $=$ disinhibition, Irrit./lab. = irritability/lability, Ab. mot. act. = aberrant motor activity, Sleep/night. = sleep/nighttime disorder, and App./eat. dis. = appetite/eating disorder.

Figure 3. Mean scores and standard errors of caregiver's distress before and after intervention for each Neuropsychiatric Inventory item.

sion (2.00 vs. 1.00$), t(10)=3.03, p=.01$, and $d=.66$, aberrant motor activity (3.00 vs. 1.82$), t(10)=2.36, p=.04$, and $d=$ .78 , and disinhibition ( 1.36 vs. 0.64$), t(10)=2.60, p=.03$, and $d=.45$. Other comparisons tended to change after the AAT intervention, with a decrease in distress associated with delusions (2.64 vs. 1.64$), t(10)=1.85, p=.09$, and $d=.46$, and with euphoria (1.18 vs. 0.18 ), $t(10)=1.98, p=.08$, and $d=.86$. All other $p$ values were greater than .10 .

\section{Descriptive Analyses at the Individual Level}

As mentioned above, the data of two participants (one man and one woman) were not included in the group analysis because of their infrequent participation in the intervention. A descriptive analysis of these two participants' data is presented here to offer more insight into the reasons for their infrequent participation in AAT. Mean scores for each period and category are presented in parentheses as follows: (Period 1/Period 2/Period 3/Period 4). The male participant missed several sessions because of a lack of interest in the dog. In general, this patient was strongly apathetic and often slept in the afternoon; his interest in the AAT intervention did not improve with time, as also revealed by his scores on interaction with the dog $(1.25 / 0 / 0 / 0)$ and his participation during the session $(0 / 0.5 / 1 / 0.7)$. Consistently, no positive affect was reported $(0$ for each of the four periods) whereas negative affect (i.e., agitation) was sometimes reported $(0.5 / 0.5 / 0 / 2)$. In contrast, the female participant was very positive about this intervention and participated frequently, but unfortunately her health strongly deteriorated and she was thus not able to attend the AAT sessions until the end of the intervention. Her attendance was marked by high levels of positive affect (1.8/3.6/3.7/-) and low levels of negative affect $(1 / 2 / 1 /-)$ as well as by high levels of interaction with the $\operatorname{dog}(3.2 / 3.2 / 3.3 /-)$ and participation during the sessions $(3 / 3.2 / 3.7 /-)$. All in all, lack of interest/apathy and poor health were the reasons for low adherence to the AAT intervention.

As to the other participants, some observations were ob- tained for a more qualitative investigation. First, and as highly understandable for older adults with severe Alzheimer's disease or a related dementia, their behaviors fluctuated strongly from session to session. The participants interacted with the dog spontaneously in some sessions, but showed no interest in the dog in other sessions. This type of behavior appeared more often in participants with high levels of agitation or anxiety. Another observation was that, for some participants, interactions with the dog were only nonphysical (i.e., gazes, smiles, questions addressed to and discussion about the dog). Contrary to our expectations, having owned a dog in the past was not a strong determinant of level of interest and participation in this AAT intervention. Participants who had never owned a dog numbered among the most involved and interested participants. This was perhaps due to the possibility that those who had had dogs, those were "working dogs" (e.g., guard dogs, hunting dogs, sheep dogs) and not family pets. Last but not least, some patients' relatives enjoyed visiting during the AAT sessions, and the dog appeared to facilitate the exchanges between them by being a topic of discussion or the object of greetings and pats from the relatives as well. In fact, one of the participants was quite indifferent to the dog, but her husband petted it a lot, and the dog became the center of their exchanges.

\section{Discussion}

The present pilot assessed the efficacy of an AAT program implemented weekly in a nursing home to reduce neuropsychiatric symptoms in older adults with medium to severe dementia. In addition, participants' behavior was observed during the different sessions and throughout the course of the AAT.

The effect of the AAT intervention on participants' behavior during the sessions appears to have been progressive, but not linear: Assessed in the four categories, the participants' behav- 
ior continued to change with increasing number of sessions. In the present study, the observed behaviors were assigned to one of four categories (negative affect, positive affect, interaction with the dog, and participation during the sessions); changes, although nonlinear, were observed in all four categories. The increase in negative affect over time was an unexpected, unpredicted result that may have been due to the fact that AAT sessions were conducted in a group and not in an individual setting, and that, before the intervention, most of participants had presented with high levels of anxiety and/or agitation. Some anxiety and aggressiveness may have been provoked by the group setting. Future investigations should focus more on the possible generation of anxiety and aggressiveness. On the other hand, the level of negative affect remained low and it seems reasonable to postulate that negative affect was offset by the concomitant increase in positive affect. Our analysis of the various AAT session periods revealed that the increase in positive affect occurred during the initial sessions (i.e., between Periods 1 and 2) and remained stable thereafter. However, the participants' amount of interaction with the dog tended to decline over time. This may have resulted from a gradual loss of interest in the animal used in this study; one may be able to lessen the loss of interest by introducing other types of animals (e.g., birds, rabbits) to increase variety. Another possible explanation for the participants' declining amount of interaction with the dog is that participants were increasingly interacting more with the other people (i.e., therapist, other participants, their relatives) who were present at the sessions and expressed more thoughts about the situation (e.g., memories) to them. This interpretation is congruent with the observation that participation behaviors increased between the first and final (i.e., 19th) session. This is a particularly pleasing result given that one of the goals of AAT is to facilitate interpersonal exchange between patient and therapist as well as other participants during group-based AAT. This aspect is also highlighted by the qualitative observations of interactions between participants and their relatives (in cases in which relatives were present during the sessions). All in all, these initial observations of patients' behavior during and across sessions provide us with a greater understanding of the underlying processes of AAT. In future studies, it will be important to investigate individual characteristics linked to AAT. Our data suggest that previous ownership of an animal does not necessarily result in active participation in or adherence to the AAT intervention.

In the present study, we also investigated the effect of AAT on the neuropsychiatric symptoms of dementia. Our results not only point out the beneficial effect of AAT on the expression of neuropsychiatric symptoms, they especially shed light on the perceived consequences of these symptoms by caregivers. Indeed, although we only observed a slightly decreasing tendency in the participants' NPI scores for depression, euphoria, and aberrant motor activity, we found significant decreases in the caregivers' ratings of distress linked to the neuropsychiatric symptoms of depression, aberrant motor activity, and disinhi- bition along with decreases in delusion and euphoria scores. An unexpected result was the increase in the score for hallucinations (influenced largely by the score of 2 of the 11 participants), whereas this increase was not significant on the caregiver distress scores related to patients' hallucinations.

Like the majority of studies on AAT (Filan \& LlewellynJones, 2006), the present study suffers from a small sample size, which may have reduced the statistical power and thus our ability to detect more statistically significant effects (Bernabei et al., 2013). This limitation is due to the context of the research, that is, an empirical examination of a psychological intervention implemented in an Alzheimer's disease/dementia care unit in a nursing home. This type of specialized care unit typically has a small inpatient capacity. Moreover, given that all of the residents were included in the initial sample, it was not possible for us to create a control group. Because of the lack of a control group, we cannot exclude the possibility that other variables besides the implemented AAT might explain the observed effects (Vrbanac et al., 2013). In the present study, conducted over a period of five months, a control group would have allowed us to control possible environmental artefacts as well as a possible time-related increase in neuropsychiatric symptoms due to the aggravation of dementia severity. Indeed, an increase in agitation and depression in the control group has been observed over a 10-week period (Majić, Gutzmann, Heinz, Lang, \& Rapp, 2013), and it is possible that the observed benefits of the AAT were reduced by the aggravation of dementia over time. In the future, a multisite intervention in other nursing homes and the use of a waiting-list control group would allow us to control these aspects and to increase the sample size.

The decreases we observed in several neuropsychiatric symptoms are in line with the existing literature (Bernabei et al., 2013; Virués-Ortega et al., 2012) and support the use of AAT as a nonpharmacological intervention aimed at the reduction of neuropsychiatric symptoms in dementia. Moreover, the observation of behaviors during the therapy sessions provides us with some insight on the process of AAT effects and on how to improve AAT efficiency. In future investigations, it would be interesting to explore whether some format modifications (e.g., frequency and duration, individual rather than group sessions) result in a lower level of negative affect without undesirable effects on other components like positive affect and interaction. Our results suggest that AAT has to be implemented on a regular and permanent basis, and this is in line with the finding that the long-term effects of AAT after sessions end is uncertain (Bernabei et al., 2013), probably due to the neurodegenerative nature of Alzheimer's disease. Moreover, it is impossible to reject the possibility that the ratings were unconsciously influenced by the raters. Thus, in future studies, several independent raters blind to the research goal should be used to limit this risk.

Finally, since positive changes in neuropsychiatric symptoms were larger on distress than on total score estimations, AAT could help the nursing staff deal with the difficulties asso- 
ciated with behavioral alterations. Data are still quite scarce, but Bibbo (2013) suggested that AAT may help to alleviate workplace stress and improve staff member mood without creating extra work. As for the processes of this benefit, AAT interventions appeared to facilitate exchanges and communication between patients and nursing staff (Richeson, 2003), which could translate into AAT benefits for patients. Moreover, Zilcha-Mano et al. (2011) suggested that, during AAT, the pet can offer a safe haven and secure base for the therapist, especially in challenging or complicated situations (see also Tribet et al., 2008). Consequently, it appears promising for future investigations to examine the influence of AAT, or more specifically pets, on the therapist and the formal and informal caregivers.

\section{Conclusion}

Even if the present pilot study revealed only a modest effect of AAT on NPI total scores, several significant improvements were observed in the caregivers' distress scores. This result underscores the effective, beneficial influence of AAT on the consequences of neuropsychiatric symptoms as perceived by caregivers. Accordingly, these results speak for the use of regular and long-term AAT interventions as a nonpharmacological alternative for neuropsychiatric symptom reduction. Further investigation is necessary to define the specific format, frequency, and duration of AAT that produces the most positive effects on AD patients' behaviors.

\section{References}

Ballard, C., \& Corbett, A. (2010). Management of neuropsychiatric symptoms in people with dementia. CNS Drugs, 24, 729-739. doi 10.2165/11319240-000000000-00000

Bernabei, V., De Ronchi, D., La Ferla, T., Moretti, F., Tonelli, L., Ferrari, B., ... Atti, A. R. (2013). Animal-assisted interventions for elderly patients affected by dementia or psychiatric disorders: A review. Journal of Psychiatric Research, 47, 762-773. doi 10.1016/j.jpsychires.2012.12.014

Bibbo, J. (2013). Staff members' perceptions of an animal-assisted activity. Oncology Nursing Forum, 40, E320-E326. doi 10.1188/13.ONF.E320-E326

Crowley-Robinson, P., Fenwick, D. C., \& Blackshaw, J. K. (1996). A long-term study of elderly people in nursing homes with visiting and resident dogs. Applied Animal Behavior Science, 47, 137-148. doi 10.1016/0168-1591(95)01017-3

Cummings, J. L., \& McPherson, S. (2001). Neuropsychiatric assessment of Alzheimer's disease and related dementias. Aging Clinical and Experimental Research, 13, 240-246. doi 10.1007/ BF03351482

Cummings, J. L., Mega, M., Gray, K., Rosenberg-Thompson, S., Carusi, D. A., \& Gornbein, J. (1994). The Neuropsychiatric Inventory:
Comprehensive assessment of psychopathology in dementia. Neurology, 44, 2308-2314. doi 10.1212/WNL.44.12.2308

Epple, D. M. (2002). Senile dementia of the Alzheimer type. Clinical Social Work Journal, 30, 95-110. doi 10.1023/A:1014230529175

Filan, S. L., \& Llewellyn-Jones, R. H. (2006). Animal-assisted therapy for dementia: A review of the literature. International Psychogeriatrics, 18, 597-611. doi 10.1017/S1041610206003322

Fine, A. H. (2010). Handbook on animal-assisted therapy: Theoretical foundations and guidelines for practice (3rd ed.). San Diego, CA: Elsevier.

Kalafat, M., Hugonot-Diener, L., \& Poitrenaud, J. (2003). Standardisation et étalonnage français du Mini Mental State (MMS) version GRECO [French standardization of the Mini Mental State (MMS), GRECO's version]. Revue de Neuropsychologie, 13, 209-236.

Kruger, K. A., \& Serpell, J. A. (2006). Animal-assisted interventions in mental health: Definitions and theoretical foundations. In A. H. Fine (Ed.), Handbook on animal-assisted therapy: Theoretical foundations and guidelines for practice (pp. 21-38). San Diego, CA: Elsevier.

Levinson, B. M. (1969). Pet-oriented child psychotherapy. Springfield, IL: CC Thomas.

Majić, T., Gutzmann, H., Heinz, A., Lang, U. E., \& Rapp, M. A. (2013). Animal-assisted therapy and agitation and depression in nursing home residents with dementia: A matched case-control trial. The American Journal of Geriatric Psychiatry, 21, 1052-1059. doi 10.1016/j.jagp.2013.03.004

Motomura, N., Yagi, T., \& Ohyama, H. (2004). Animal assisted therapy for people with dementia. Psychogeriatrics, 4, 40-42. doi $10.1111 / j .1479-8301.2004 .00062 . x$

Pollock, B. G., \& Mulsant, B. H. (2011). Between Scylla and Charybdis: Antipsychotic and other psychotropic medications in older nursing home residents. Canadian Medical Association Journal, 183, 778-779. doi 10.1503/cmaj.110348

Richeson, N. E. (2003). Effects of animal-assisted therapy on agitated behaviors and social interactions of older adults with dementia. American Journal of Alzheimer's Disease and Other Dementias, 18, 353-358. doi 10.1177/153331750301800610

Selbaek, G., Engedal, K., \& Bergh, S. (2013). The prevalence and course of neuropsychiatric symptoms in nursing home patients with dementia: A systematic review. Journal of the American Medical Directors Association, 14, 161-169. doi 10.1016/j.jamda.2012.09. 027

Sisco, F., Taurel, M., Lafont, V., Bertogliati, C., Baudu, C., Girodana, J.Y., ... Robert, P. (2000). Les troubles du comportement chez les sujets déments en institution: Evaluation à partir de l'inventaire neuropsychiatrique pour les équipes soignantes [Behavior disorders in elderly dementia: Neuropsychiatric Inventory for Health Staff]. L'Année Gérontologique, 14, 151-171.

Tribet, J., Boucharlat, M., \& Myslinski, M. (2008). Le soutien psychologique assisté par l'animal à des personnes atteintes de pathologies démentielles sévères [Animal-assisted therapy for people suffering from severe dementia]. L'Encéphale, 34, 183-186. doi 10.1016/j.encep.2007.01.006

van der Ploeg, E. S., Mbakile, T., Genovesi, S., \& O'Connor, D. W. (2012). The potential of volunteers to implement nonpharmacological interventions to reduce agitation associated with dementia in nursing home residents. International Psychogeriatrics, 24, 1790-1797. doi 10.1017/S1041610212000798

Virués-Ortega, J., Pastor-Barriuso, R., Castellote, J. M., Población, A., \& de Pedro-Cuesta, J. (2012). Effect of animal-assisted therapy on the psychological and functional status of elderly populations and patients with psychiatric disorders: A meta-analysis. Health Psychology Review, 6, 197-221. doi 10.1080/17437199.2010. 534965 Vrbanac, Z., Zecević, I., Ljubić, M., Belić, M., Stanin, D., Bottegaro, N. B., ... Zubčić, D. (2013). Animal assisted therapy and perception of loneliness in geriatric nursing home residents. Collegium Antropologicum, 37, 973-976. 
Walsh, P. G., Mertin, P. G., Verlander, D. F., \& Pollard, C. F. (1995). The effects of a "pets as therapy" dog on persons with dementia in a psychiatric ward. Australian Occupational Therapy Journal, 42, 161-166. doi 10.1111/j.1440-1630.1995.tb01331.x

Wood, S. A., Cummings, J. L., Barclay, T., Hsu, M.-A., Allahyar, M., \& Schnelle, J. F. (1999). Assessing the impact of neuropsychiatric symptoms on distress in professional caregivers. Aging \& Mental Health, 3, 241-245. doi 10.1080/13607869956208

Wood, S., Cummings, J. L., Hsu, M.-A., Barclay, T., Wheatley, M. V., Yarema, K. T., \& Schnelle, J. F. (2000). The use of the Neuropsychiatric Inventory in nursing home residents: Characterization and measurement. The American Journal of Geriatric Psychiatry, 8, 75-83. doi 10.1097/00019442-200002000-00010

Zilcha-Mano, S., Mikulincer, M., \& Shaver, P. R. (2011). Pet in the therapy room: An attachment perspective on animal-assisted therapy. Attachment \& Human Development, 13, 541-561. doi $10.1080 / 14616734.2011 .608987$

\section{Isabelle Tournier}

\section{INSIDE}

University of Luxembourg

Maison des Sciences Humaines

11 porte des sciences

4366 Esch-sur-Alzette

Luxembourg

isabelle.tournier@uni.lu 